\title{
Ultrastructural Localization of the Serotonin Transporter in Limbic and Motor Compartments of the Nucleus Accumbens
}

\author{
Virginia M. Pickel and June Chan \\ Department of Neurology and Neuroscience, Weill Medical College of Cornell University, New York, New York 10021
}

\begin{abstract}
Extracellular levels of serotonin [5-hydroxytryptamine (5-HT)] in the nucleus accumbens (NAc) can influence both cognitive and motor functions involving extensive connections with the frontal cortex. The 5-HT levels reflect vesicular release and plasmalemmal reuptake through the serotonin transporter (SERT). We used electron microscopic immunocytochemistry to determine the sites for SERT activation in the limbic shell and motorassociated core of the rat NAc. Of the SERT-immunoreactive profiles in each region, $>90 \%$ were serotonergic axons and axon terminals; the remainder were nonserotonergic dendrites and glia. Axonal SERT immunogold labeling was seen mainly at nonsynaptic sites on plasma membranes and often near 5-HTcontaining large dense core vesicles (DCVs). SERT-labeled axonal profiles were larger and had a higher numerical density in the shell versus the core but showed no regional differences in
\end{abstract}

their content of SERT immunogold particles. In contrast, immunoreactive dendrites had a lower numerical density in the shell than in the core. SERT labeling in dendrites was localized to segments of plasma membrane near synaptic contacts from unlabeled terminals and/or dendritic appositions. Our results suggest that in the NAc (1) reuptake into serotonergic axons is most efficient after exocytotic release from DCVs, and (2) increased 5-HT release without concomitant increase in SERT expression in individual axons may contribute to higher extracellular levels of serotonin in the shell versus the core. These findings also indicate that SERT may play a minor substratedependent role in serotonin uptake or channel activity in selective nonserotonergic neurons and glia in the NAc.

Key words: dense core vesicle; presynaptic; nonserotonergic dendrites; astrocyte; volume transmission; depression
Vesicular release of serotonin (Gobbi et al., 1998) is accompanied by plasmalemmal reuptake into serotonergic neurons through the serotonin transporter (SERT) (Qian et al., 1995; Schroeter et al., 1997). Thus, SERT mRNA is abundant in serotonergic raphe neurons (Austin et al., 1994; Charnay et al., 1996), many of which project to the nucleus accumbens (NAc) (Van Bockstaele et al., 1993). The NAc is part of the ventral striatum in which low extracellular serotonin levels have been correlated with high anxiety in rats (Schwarting et al., 1998). Anxiety also is seen often in depressed patients, including those showing symptoms of schizophrenia (Nutt, 1995; Joyce et al., 1997). Drugs such as fluoxetine that increase extracellular levels of 5-hydroxytryptamine (5-HT) via binding to SERT are effective antidepressants, suggesting that the functional sites for SERT activation in the NAc and related brain regions are critical for their therapeutic effectiveness (for review, see Schloss and Williams, 1998; Staley et al., 1998).

Serotonergic afferents to the NAc are distributed more prominently in the shell than in the core (Van Bockstaele and Pickel, 1993). Neurons in the shell region also express higher levels of 5-HT ${ }_{2 \mathrm{a}}$ receptor mRNA (Mijnster et al., 1997), suggesting that the shell may play a more major role than the core in functions that are ascribed to serotonin in the NAc. This conclusion is consistent with the extensive projection to the shell from the prefrontal cortex (Sesack and Pickel, 1992) that, together with the

Received April 6, 1999; revised June 21, 1999; accepted June 23, 1999.

V.M.P. receives salary support from a Career Award from the National Institute of Mental Health (NIMH) Grant MH00078 and research support from National Institute on Drug Abuse Grant DA04600 and NIMH Grant MH40342. We thank Dr. Adena L. Svingos for helpful critical commentary.

Correspondence should be addressed to Dr. Virginia M. Pickel, Department of Neurology and Neuroscience, Cornell University Medical College, 411 East 69th Street, KB 410, New York, NY 10021.

Copyright (C) 1999 Society for Neuroscience $0270-6474 / 99 / 197356-11 \$ 05.00 / 0$
NAc, receives major input from the amygdala and other limbic structures involved in cognitive and motivational functions (Brog et al., 1993; Groenewegen et al., 1997).

The subcellular distribution of SERT has not been examined in either the shell or the core of the NAc, but in other regions SERT is localized to nonsynaptic sites in serotonergic axons (Zhou et al., 1998). This distribution is comparable to that of the plasmalemmal dopamine transporter (DAT), which is present in lower levels in individual axons in the NAc shell than in the core (Nirenberg et al., 1997). Low levels of DAT expression in these axons are likely to contribute to the higher extracellular levels of dopamine in the NAc shell versus core (Jones et al., 1996). Whether there are also regional differences in levels of SERT in the NAc is not known but might be expected, because psychostimulants that bind SERT produce a more dramatic increase in extracellular levels of serotonin in the caudal shell than in the core (Heidbreder and Feldon, 1998). To determine the functional sites for SERT activation and potential regional differences in SERT expression in the NAc, we quantitatively compared the electron microscopic immunocytochemical localization of antipeptide antisera against SERT in the shell and core of rat brain.

\section{MATERIALS AND METHODS}

Antisera source. Antipeptide $\left(\mathrm{C}_{611-630}\right.$ and $\left.\mathrm{E}_{387-401}\right)$ and anti-fusion protein antisera $\left(\mathrm{N}_{1-85}\right)$ were raised against epitopes of the cloned SERT that are less well conserved among $\mathrm{Na}^{+} / \mathrm{Cl}^{-}$cotransporters (Qian et al., 1995). The affinity-purified goat polyclonal $\mathrm{C}_{611-630}$ antiserum was obtained from Santa Cruz Biotechnology (catalog number SC-1458; Santa Cruz, CA). The affinity-purified rabbit polyclonal $\mathrm{E}_{387-401}$ was obtained from Chemicon (catalog number AB1594P; Temecula, CA). This antiserum was shown by ELISA to recognize at 1:100 K 50-100 ng of SERT peptide (catalog number AG297; Chemicon). The mouse monoclonal antiserum against glutathione $S$-transferase (GST) fusion protein $\mathrm{N}_{1-85}$ was obtained from Chemicon (catalog number MAB 1564). In addition, 
a rat antiserum that was raised against a glutaraldehyde conjugate of 5-HT as described by Steinbusch et al. (1978) was obtained from Accurate Chemicals (catalog number MAS-055b; Westbury, NY).

Animals and fixation procedure. Adult male Sprague Dawley rats (250$400 \mathrm{gm}$ ) were purchased from Hilltop Laboratory Animals (Scottsdale, PA). The experimental protocol for the use of these animals strictly conforms with National Institutes of Health Guidelines for the Care and Use of Laboratory Animals and was approved by the Institutional Animal Care and Use Committee of Weill Medical College of Cornell University. The rats were anesthetized with sodium pentobarbital $(100 \mathrm{mg} / \mathrm{kg}$, i.p.) and perfused through the aortic arch with (1) $30-50 \mathrm{ml}$ of $3.75 \%$ acrolein in $2 \%$ paraformaldehyde or (2) $200 \mathrm{ml}$ of $0.2 \%$ glutaraldehyde and $4 \%$ paraformaldehyde. These fixative solutions were prepared in 0.1 $\mathrm{M}$ phosphate buffer (PB) at $\mathrm{pH}$ 7.4. The brains were removed from the cranium and cut into 2-4 $\mathrm{mm}$ slices of tissue, including forebrain regions that contained the NAc. These were sectioned at a thickness of $40 \mu \mathrm{m}$ on a vibrating microtome and rapidly freeze-thawed to enhance penetration. For this, free-floating sections were cryoprotected in $25 \%$ sucrose and $3.5 \%$ glycerol in $\mathrm{PB}$, rapidly frozen in Freon and liquid nitrogen, and thawed in room temperature PB. These sections were processed for immunocytochemical labeling by the use of peroxidase and/or gold markers (Leranth and Pickel, 1989).

Immunoperoxidase labeling. For immunoperoxidase labeling the freefloating coronal sections through the NAc were incubated for $24 \mathrm{hr}$ at room temperature or for $48 \mathrm{hr}$ at $4^{\circ} \mathrm{C}$ in the three primary anti-SERT antisera. Dilutions of the three antisera were prepared in $0.1 \% \mathrm{BSA}$ in Tris-saline at 1:5000-20,000, 1:100 or 1:1000, and 1:1000, respectively. After this incubation the sections were rinsed and placed for $30 \mathrm{~min}$ in biotinylated anti-IgGs (1:400) corresponding to the species of the primary antiserum and avidin-biotin peroxidase complex (Vector Elite Kit, Vector Labs, Burlingame, CA). The bound peroxidase was identified by reaction of the sections for $6 \mathrm{~min}$ in 3,3' diaminobenzidine (Aldrich Chemicals, Milwaukee, WI) and hydrogen peroxide. For light microscopy the sections were mounted on glass slides, dehydrated, coverslipped, and examined on a Nikon microscope (Nikon, Garden City, NY). For electron microscopy the sections were post-fixed in $2 \%$ osmium tetroxide in $0.1 \mathrm{M}$ phosphate buffer, dehydrated, and flat-embedded between two pieces of Aclar plastic. Ultrathin sections from the NAc core and shell regions were collected onto grids with an LKB ultratome (LKB-Wallac, Gaithersburg, MD). The sections on grids were counter-stained with Reynold's lead citrate and uranyl acetate and examined with a Philips CM-10 electron microscope (Mahwah, NJ).

Immunogold-silver labeling. For immunogold-silver labeling of SERT the sections that were prepared as described above were incubated in the goat $\mathrm{C}_{611-630}$ antiserum at a dilution of 1:1000 or 1:5000. After $24 \mathrm{hr}$ these sections were rinsed in Tris buffer and placed for $30 \mathrm{~min}$ in a 1:50 dilution of rabbit anti-goat IgG with bound $1 \mathrm{~nm}$ colloidal gold (Amersham, Arlington Heights, IL). The gold particles were enlarged for microscopic examination by reaction for $6 \mathrm{~min}$ at room temperature in a silver solution from the IntenS-EM kit (Amersham).

For dual labeling the same protocol was used except that (1) the goat $\mathrm{C}_{611-630}$ SERT antiserum was combined with rat monoclonal antiserum against 5-HT at 1:400 dilution, and (2) the sections were processed by using goat anti-rat biotinylated IgG (1:400) and the Vector Elite ABC Kit. Then these sections were processed for immunogold detection of the SERT antiserum, as previously described (Chan et al., 1990). The labeled sections were prepared for light and electron microscopic examination in the same manner as for the peroxidase labeled sections (see above).

Adsorption controls. As controls for specificity the sections of tissue were processed for immunoperoxidase or immunogold labeling, with the omission of primary antisera or with primary antiserum that had been preadsorbed with the antigenic peptide. The adsorption control was prepared only for the $\mathrm{C}_{611-630}$ antiserum, which was used for the major analysis. For this, $12 \mu \mathrm{l}$ of a solution containing $100 \mu \mathrm{g}$ of the C-terminal peptide in $0.5 \mathrm{ml}$ of PBS (catalog number SC-1458 P; Santa Cruz Biotechnology) was placed in $2.0 \mathrm{ml}$ of the primary $\mathrm{C}_{611-630}$ antiserum at a dilution of 1:1000. This antiserum, together with $2.0 \mathrm{ml}$ of the same antiserum without the corresponding peptide, was placed on a shaker at $4^{\circ} \mathrm{C}$ for $24 \mathrm{hr}$ and then centrifuged for $30 \mathrm{~min}$ at maximum speed on a Beckman Microfuge (Fullerton, CA). Sections then were processed for immunoperoxidase or immunogold labeling by using the adsorbed and nonadsorbed antisera and were examined by light and electron microscopy.

Sampling and labeling criteria. Electron microscopic images were examined from thin sections collected from the outer surface of vibratome sections through the NAc shell and core at a level $0.7-1.8 \mathrm{~mm}$ anterior to Bregma, according to the atlas of the rat brain (Paxinos and Watson, 1986). For quantitative comparison these thin sections were collected from shell and core regions within the same vibratome sections. A profile was considered to be immunogold-labeled selectively when one or more gold particles were seen in contact with the plasmalemma or limiting membranes of cytoplasmic organelles. This was possible because of the low background labeling that was seen under the labeling conditions in this study and the preferential localization of SERT to plasma membranes.

Compartmental distribution of SERT labeling. Sections that were processed by immunoperoxidase labeling were used for shell and core comparison of the cellular distribution of SERT in neuronal and glial compartments. The labeled profiles were examined in thin sections that were taken from one to three vibratome sections in four animals. All profiles in single sections through each region were stored at a magnification of $7900 \times$, using specimen relocation software on a CM-10 electron microscope. Subsequently, each profile was examined at higher magnification and binned into categories of small unmyelinated axons, axon terminals, dendrites, dendritic spines, or glial processes (Peters et al., 1991). Those labeled profiles that were not clearly identifiable in any of these groups were placed in a group of nonidentifiable or unknown structures.

Numerical density of SERT-labeled profiles. The numerical density of profiles that contained peroxidase labeling for SERT in the NAc shell and core was estimated by using a physical disector (Sterio, 1984). For this, serial ultrathin sections were collected and photographed at a magnification of $8900 \times$. These micrographs were enlarged, and a $4 \times 4$ $\mu \mathrm{m}$ square was drawn on each printed micrograph within each series of serial sections. The number of labeled profiles was counted in a look-up plane if they did not appear in the reference plane. The volume of the disector was calculated by using the estimated thickness of a single thin section at $60 \mathrm{~nm}$. A total of 426 dissectors (238 in the NAc shell and 188 in the core) were generated from tissue sections that were derived from four animals. The numerical density for each category of SERT-labeled profiles was determined. Statistical tests to determine differences between core and shell were performed by using ANOVA with post hoc analysis and Sigma Stat software (St. Louis, MO).

Axonal distribution and content of SERT immunogold particles. Axonal profiles containing SERT immunogold labeling were stored by using specimen relocation software on the CM-10 electron microscope. The total number of immunogold-silver particles per profile was recorded and separated into categories of particles that were in contact, or not, with the plasma membrane. The area and perimeter of these profiles were measured also. These values were obtained for 400 labeled axons in the shell and 375 in the core.

Number of 5-HT-containing vesicles. The mean number of serotonincontaining DCVs in individual axons was determined in the NAc shell and core. The regional analysis was performed in thin sections that were collected from coronal vibratome sections through the NAC shell and core from five animals. The labeled DCVs were defined as electron dense vesicles having a diameter of $80-150 \mathrm{~nm}$.

Ilustrations. Micrographs that were used for illustrations were scanned on a Power Macintosh 8500/150 Computer (Apple Computers, Cupertino, CA) with an AGFA Arcus II scanner (Agfa-Gevaert, Montsel, Belgium) in combination with FotoLook (Agfa-Gevaert) and Photoshop software (version 5.0, Adobe Systems, Mountain View, CA). QuarkXPress (version 3.32; Quark, Denver, CO) and Adobe Illustrator (version 7.0; Adobe Systems) software were used to prepare and label the composite figures.

\section{RESULTS}

SERT-like immunoreactivity (SERT-LI) was localized to varicose processes that appeared by light microscopy to be more prevalent in the shell than in the core of the NAc. These were most evident when the goat $\mathrm{C}_{611-630}$ or rabbit $\mathrm{E}_{387-401}$ antisera were used but were also detectable with the mouse $\mathrm{N}_{1-85}$ antiserum. No similar labeling was seen in sections that were processed by using control sera. Electron microscopy confirmed the similarity of the labeling patterns with the three antisera and also showed that the immunoreactive processes were primarily small axons and axon terminals having a higher numerical density in the NAc shell than in the core (Fig. 1). In these axons, as well as 


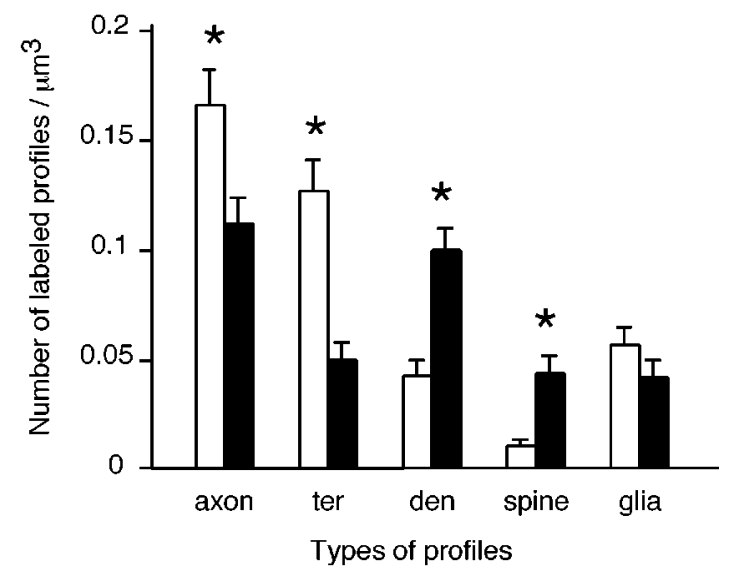

Figure 1. Bar graphs showing the numerical density of different types of SERT peroxidase-labeled profiles by using $\mathrm{C}_{611-630}$ antiserum in the NAc shell (white bars) and core (black bars). These profiles were determined from analysis of thin sections that were taken from one to three coronal vibratome sections through the NAc from four animals. The labeled small unmyelinated axons (axon), axon terminals (ter), dendrites (den), and dendritic spines (spine) show significant $\left({ }^{*}\right)$ shell and core differences in numerical density via ANOVA; $p<0.01$.

in selective dendrites and glia, SERT-LI was localized discretely to plasma membranes with each of the three antisera, whereas no similar distributions were seen in control tissue. In contrast with axons and axon terminals, dendrites and dendritic spines containing SERT-LI had lower numerical densities in the shell than in the core, whereas glia showed no significant regional variation in number (Fig. 1).

\section{SERT localization in axons and terminals}

Of the profiles that contained peroxidase labeling for SERT, $60 \%$ $(n=700)$ in the NAc shell and 61\% $(n=596)$ in the core were small $(<0.2 \mu \mathrm{m})$ unmyelinated axons. The SERT labeling in small axons appeared denser than that seen within axon terminals, irrespective of whether peroxidase or gold labeling methods were used (Figs. 2, 3). The immunoreactive axons were apposed most often by small unmyelinated axons (Fig. 2) and less frequently by dendrites or glia (Figs. 2, 3). Although most of the apposed structures were unlabeled, a few of the immunoreactive axons were seen in contact with dendrites (see Fig. 5) or glial processes that also contained SERT-LI (see Fig. 7A).

In SERT-containing axons the peroxidase reaction product often obscured cytoplasmic organelles and only occasionally was aggregated on, or near, portions of the plasma membrane (Fig. $2 A$ ). In contrast, SERT immunogold particles in small axons were localized almost exclusively to plasma membranes (Fig. 3A,B), most of which contained immunoperoxidase reaction for 5-HT in sections that were processed for dual labeling (see Fig. 2C,D). In these axons the 5-HT labeling was distributed diff usely throughout the cytoplasm or showed a more selective association with large $(80-150 \mathrm{~nm})$ vesicular organelles. These are presumed to be large DCVs, comparable to those that were seen more clearly in axons and terminals without dual labeling for 5-HT (Fig. 3B,C).

The subcellular 5-HT localization reflected, in part, the fixation conditions. In axons as well as in axon terminals a more diffuse distribution of peroxidase labeling for 5-HT was seen in glutaraldehyde-fixed tissue, whereas vesicular localization of 5-HT was seen mainly in acrolein-fixed tissue (see Figs. 2C,D, 4). Of the serotonin-immunoreactive axons and terminals that were seen in acrolein-fixed tissue, 33\% (46 of 139) in the shell and 17\%
(9 of 52) in the core contained DCVs within a single plane of section. All of these vesicles were more electron dense than similar DCVs in unlabeled terminals, indicating that they contain peroxidase immunoreactivity for 5-HT. The values were similar but lower in glutaraldehyde-fixed tissue, representing 22\% (29 of 132) of the labeled axons in the shell and 3\% (2 of 70) of those in the core.

Axon terminals comprised $29 \%$ of the SERT-labeled profiles in the NAc shell and $25 \%$ of those in the core. In each region the SERT-immunoreactive terminals were seen in a range of sizes, but those in the shell (Fig. 4A,C,D,E) usually appeared larger than in the core (Fig. $4 B$ ). The mean area of SERTimmunoreactive terminals was $0.24 \pm 0.21 \mu \mathrm{m}^{2}$ in the NAc shell and $0.18 \pm 0.15 \mu \mathrm{m}^{2}$ in the core. Despite the difference in size neither the total nor plasmalemmal labeling for SERT showed significant regional variation. Gold particles in individual axons and axon terminals had a mean number of $6.05 \pm 4.1$ in the NAc shell and $5.74 \pm 3.8$ in the core. The percentage of SERT gold particles that were in contact with the plasma membrane was $90.8 \%$ in the shell and $93.4 \%$ in the core.

There were no regional differences in the associations between SERT-labeled terminals and other neurons or in the subcellular distribution of SERT. The SERT-immunoreactive axon terminals formed symmetric synapses with large unlabeled dendrites (Fig. $4 A, B)$ as well as asymmetric synapses on smaller unlabeled dendrites and dendritic spines (see Figs. $2 A, 4 C$ ). SERT labeling was seen along plasma membranes or associated with membranes of SSVs at a distance from the synaptic specialization (see Figs. $2 A$, $4 A-C)$.

In contrast with the synaptic membrane, gold particles often were seen on plasma membranes in contact with, or near, DCVs (Figs. 3C, 4A). The DCVs in SERT-labeled terminals, like those in axons, also contained 5-HT (Fig. 4B,D,E). These vesicles and associated plasmalemmal SERT were present at appositional contacts with unlabeled dendrites and dendritic spines (Fig. 4A,B,D). The apposed dendrites were distinct from those receiving synaptic input (Fig. 4B). The storage vesicles and/or SERT plasmalemmal labeling also was apposed to unlabeled axons and terminals, some of which formed asymmetric axospinous synapses (Fig. 4E).

\section{SERT localization in nonserotonergic dendrites}

Of the SERT-immunoreactive profiles, $2 \%$ (14 of 700) in the shell and $4 \%$ (24 of 596) in the core were dendrites or dendritic spines. In sections that were processed for dual labeling, none of the dendritic profiles contained SERT and 5-HT. There were no apparent regional differences in the dendritic morphology or subcellular distribution of SERT-LI. In dendrites, SERT was localized within or near the perimeter of synaptic junctions that were formed by unlabeled terminals (Fig. 5). The labeled synaptic specializations on dendrites were symmetric, whereas those on spines were asymmetric. Many of these unlabeled terminals also formed the same types of junctions with other spines or dendrites that contained little, if any, SERT immunoreactivity (Fig. 5B,C).

SERT-LI also was seen along discrete segments of plasma membranes of one member of two apposed dendrites (Fig. 6). These dendrites did not show aggregates of vesicles and rarely received synaptic input from axon terminals within the plane of section. One example was seen in which the inner leaflets of the apposed plasma membranes were fused in a manner that has been described for dendritic gap junctions (Fig. 6A) (Peters et al., 1991). In these pairs of apposed dendrites the SERTimmunoreactive dendrites often contained only one mitochon- 

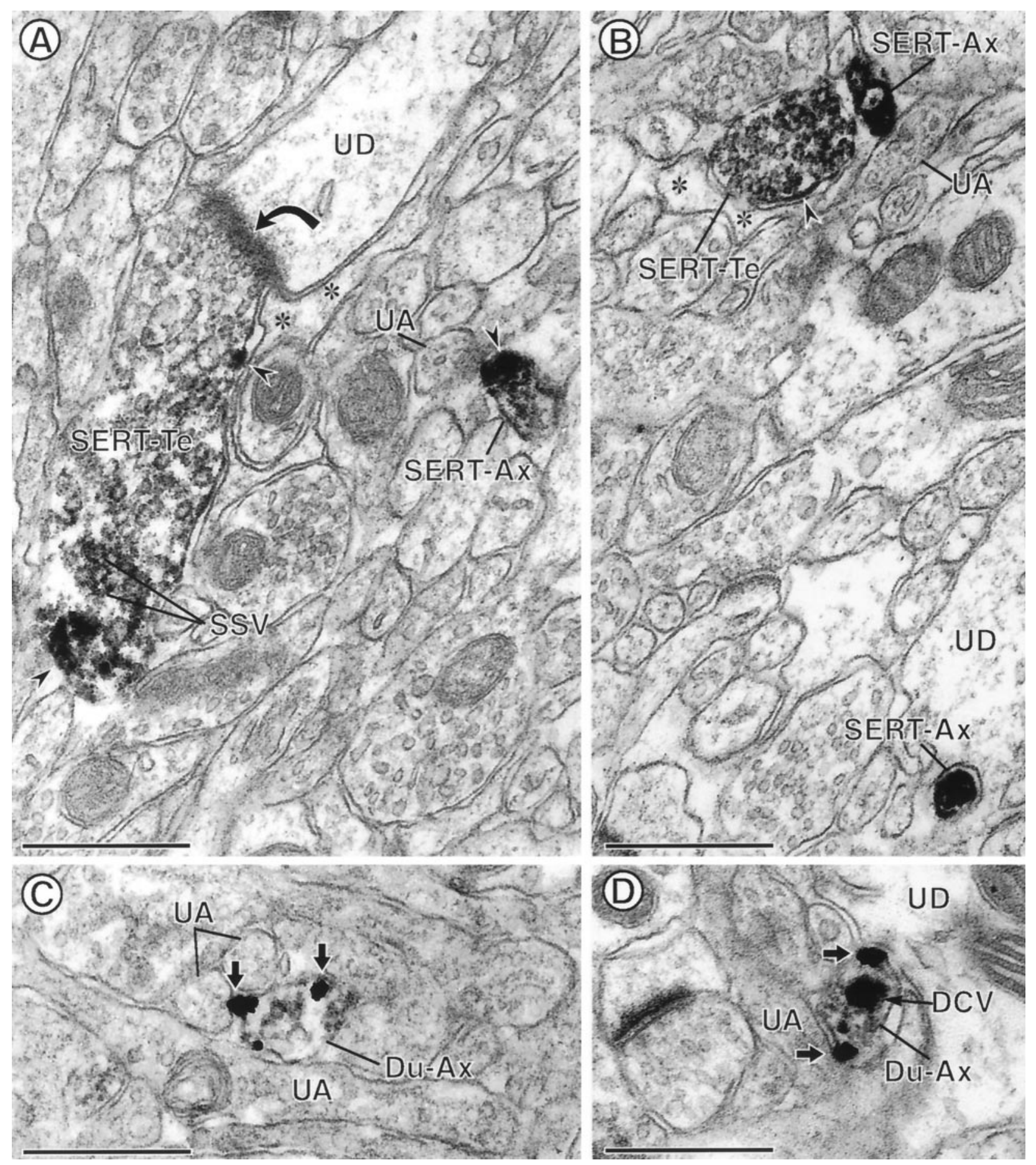

Figure 2. Electron micrographs showing immunoperoxidase localization of SERT and dual labeling for SERT and 5-HT in small axons and terminals in the NAc from acrolein-fixed rat brain. $A, B$, In the NAc core and shell, respectively, peroxidase labeling for SERT is seen in small axons (SERT- $A x)$ and terminals $(S E R T-T e)$ by using the $\mathrm{C}_{611-630}$ antiserum at 1:20,000 dilution. The peroxidase labeling is prominent on or near the plasma membranes (arrowhead) and more lightly distributed around membranes of small synaptic vesicles $(S S V s)$ at a distance from the asymmetric synapse (curved arrow) that is formed with an unlabeled dendrite $(U D)$ in $A$. The intense labeling in small axons in large part obscures their content of organelles. The SERT-labeled axons are apposed mainly by small unlabeled axons $(U A)$ or unlabeled dendrites $(U D)$. Asterisks show unlabeled glial processes. $C$, $D$, In the NAc core and shell, respectively, small unmyelinated axons are dually labeled $(D u-A x)$ for SERT and 5-HT. Diff use peroxidase labeling for 5-HT is seen within the axon, whereas immunogold SERT labeling (small arrows) is localized along the plasma membrane. In addition, a large, presumably dense core vesicle $(D C V)$ in $\mathrm{D}$ also contains peroxidase immunoreactivity. The labeled axons are apposed to unlabeled axons $(U A)$ in $C$ and to unlabeled axons and an unlabeled dendrite $(U D)$ in $D$. Scale bars, $0.5 \mu \mathrm{m}$. 

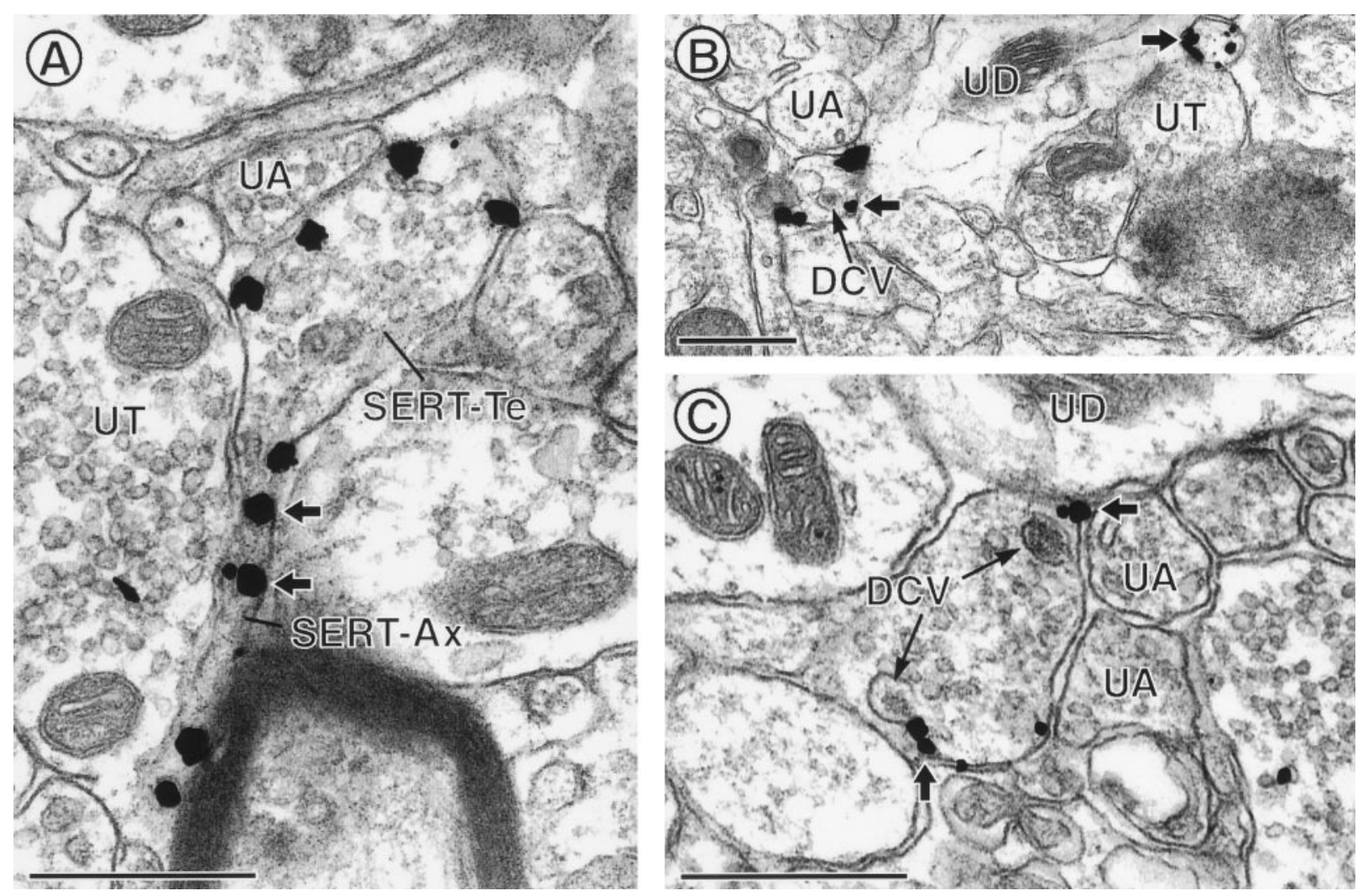

Figure 3. Immunogold-silver (arrows) localization of SERT in small axons and axon terminals in the NAc from acrolein-fixed rat brain. $A$, In the shell SERT labeling is seen in an axon (SERT-Ax), which is in continuity with a SERT-immunoreactive terminal $(S E R T$-Te) in tissue that was processed by using the $\mathrm{C}_{611-630}$ antiserum at a 1:1000 dilution. The labeled profile is apposed to an unlabeled axon $(U A)$ and terminal (UT). B, $C$, In the core SERT labeling is present in small axons and axon terminals, respectively, in tissue that was processed by using the same antiserum but at a 1:5000 dilution. Gold particles are seen along portions of the plasma membrane in contact with large dense core vesicles $(D C V s)$ that are present in a small axon $(B)$ and an axon terminal $(C)$. Contacts are seen between the labeled axons or terminals and other unlabeled axons $(U A)$, unlabeled dendrites $(U D)$ or unlabeled terminals $(U T)$. Scale bars, $0.5 \mu \mathrm{m}$.

drion, whereas the apposed dendrites contained many large mitochondria.

\section{Glial SERT distribution}

Of the observed SERT immunoperoxidase-labeled profiles, 28 of $700(4 \%)$ in the NAc shell and 24 of 596 in the core were glia. These peroxidase-labeled glial profiles were irregularly shaped, contained filaments, and formed gap junctions that are typical of astrocytic processes. The peroxidase reaction product was distributed diffusely in the cytoplasm (Fig. 7A) or localized more discretely to plasma membranes of SERT-containing glia (Fig. $7 B, C)$. In sections that were processed for immunogold-silver localization of SERT, a few gold particles also were seen within glial profiles. The number of particles was, however, usually too small for positive identification as containing SERT-LI.

SERT-immunoreactive astrocytic plasma membranes were seen at gap junctions with unlabeled glial profiles and near appositions with unlabeled axons (Fig. 7B). In addition, the labeled glial processes were apposed to unlabeled axon terminals and dendrites, particularly those at asymmetric axospinous junctions (Fig. 7A,C). The SERT-containing glia sometimes were apposed to axons that were intensely SERT-immunoreactive (Fig. $7 A$ ) but usually were located at a distance from these axons (Fig. 7C).

\section{DISCUSSION}

We have shown that, in limbic and motor compartments of the NAc, SERT is targeted mainly to axonal plasma membranes, being most prevalent near 5-HT-containing DCVs and least abundant within synaptic membrane specializations. In addition, plasmalemmal SERT was seen in dendrites of a few nonserotonergic neurons and glia in both striatal regions. These results, together with the observed regional similarities and differences in SERT expression, are discussed with respect to their impact on extracellular levels and the function of 5-HT in the NAc.

\section{Plasmalemmal SERT distribution near dense core vesicles in axons}

We often saw SERT labeling on plasma membranes near 5-HTcontaining large DCVs in axons and axon terminals. This provides ultrastructural support for the earlier suggestion that SERT is targeted to those portions of the plasma membrane near major storage and release sites (Blakely et al., 1998). Large DCVs have been shown to contain serotonin (Pelletier et al., 1981) and also to express higher levels of the vesicular monoamine transporter 2 (VMAT2) than SSVs (Nirenberg et al., 1995). The differential expression of VMAT2 in large vesicles and the enrichment of SERT on plasma membranes apposed to DCVs may reflect their greater capacity for transmitter storage and release as compared with SSVs (Bruns and Jahn, 1995).

Serotonin-containing DCVs have an ectopic distribution around the perimeter of axon terminals, suggesting the involvement of the DCVs in nondirected transmitter release (Liem and Copray, 1996). The proximity of these vesicles to SERTimmunoreactive plasma membranes may limit extracellular dif- 

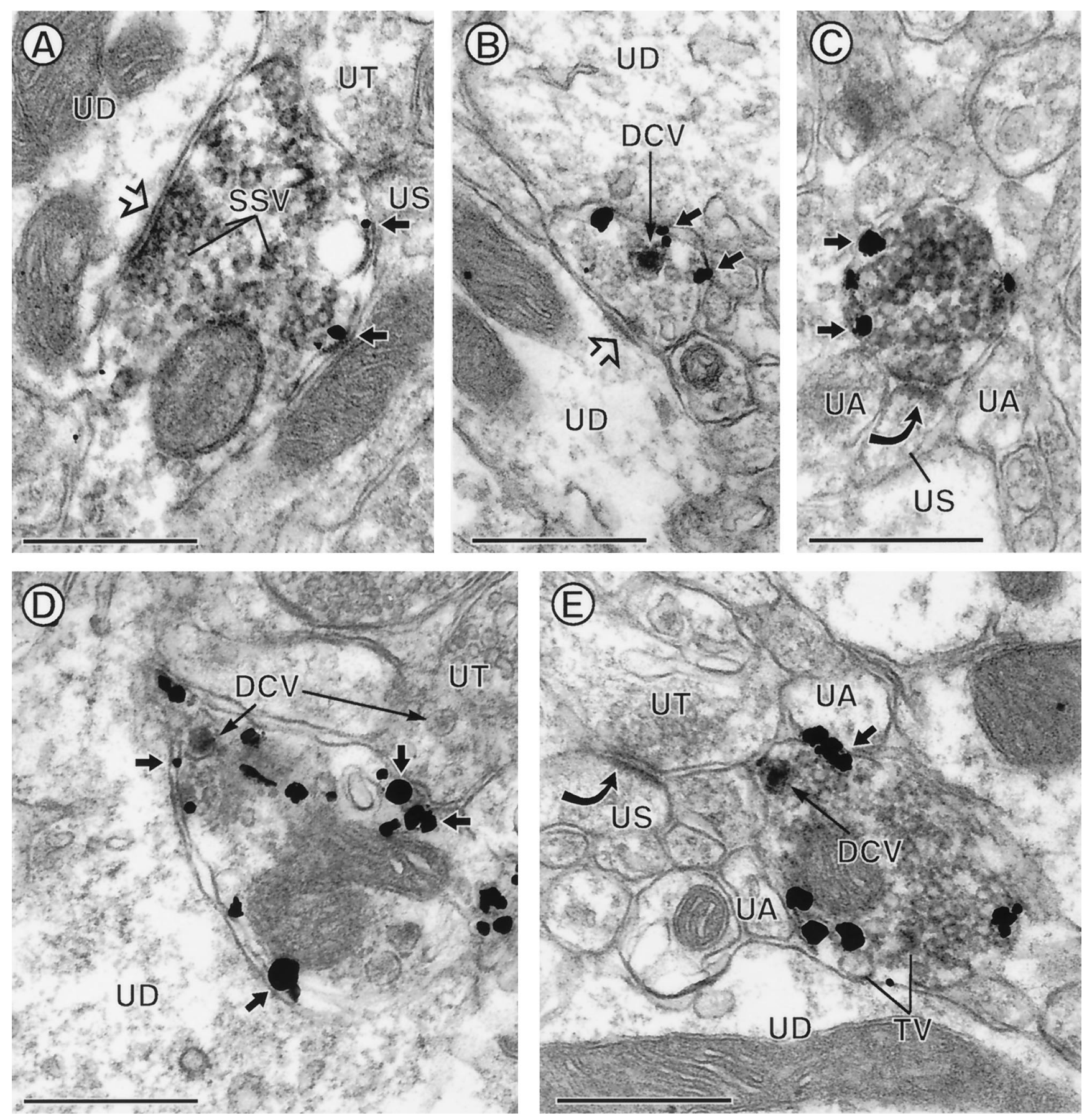

Figure 4. Dual labeling for SERT and 5-HT in axon terminals within the NAc from brain tissue that was fixed by using glutaraldehyde $(A, C)$ or acrolein $(B, D, E)$. Immunogold labeling for SERT (small arrows) is seen along portions of the plasma membrane (1) away from symmetric axodendritic (open arrowhead in $A$ from the shell and in $B$ from the core) and asymmetric axospinous (curved filled arrow in $C$ from the shell) synapses and (2) near appositional contacts with unlabeled dendrites $(U D)$ and unlabeled terminals $(U T)$ in the shell $(D, E)$. The peroxidase labeling appears as a diffuse precipitate surrounding small synaptic vesicles ( $S S V)$, as seen in $A$, or more discretely localized to membranes of large dense core vesicles ( $D C V s)$ as seen in $B, D$, and $\mathrm{E}$. The gold particles are prevalent near DCVs that contact the plasma membrane at appositional contacts with unlabeled dendrites and terminals. $E$, The apposed unlabeled terminal forms an asymmetric synapse (curved arrow) with an unlabeled spine (US). Tubulovesicular organelles $(T V)$ also are seen near the plasma membrane and contacted by gold particles. Scale bars, $0.5 \mu \mathrm{m}$.

fusion, however, to only those neuronal and/or glial profiles that are near release sites. In the present study the DCVs and associated SERT-immunoreactive plasma membranes often were apposed to unlabeled small axons and axon terminals, some of which formed asymmetric axodendritic synapses that are typical of glutamatergic terminals (Gundersen et al., 1996). Activation of $5-\mathrm{HT}_{1 \mathrm{~B}}$ serotonin receptors also is known to modulate glutamate release presynaptically (Muramatsu et al., 1998). Because many of the glutamatergic afferents in the shell of the NAc are derived from the prefrontal cortex (Sesack and Pickel, 1992), these may be 

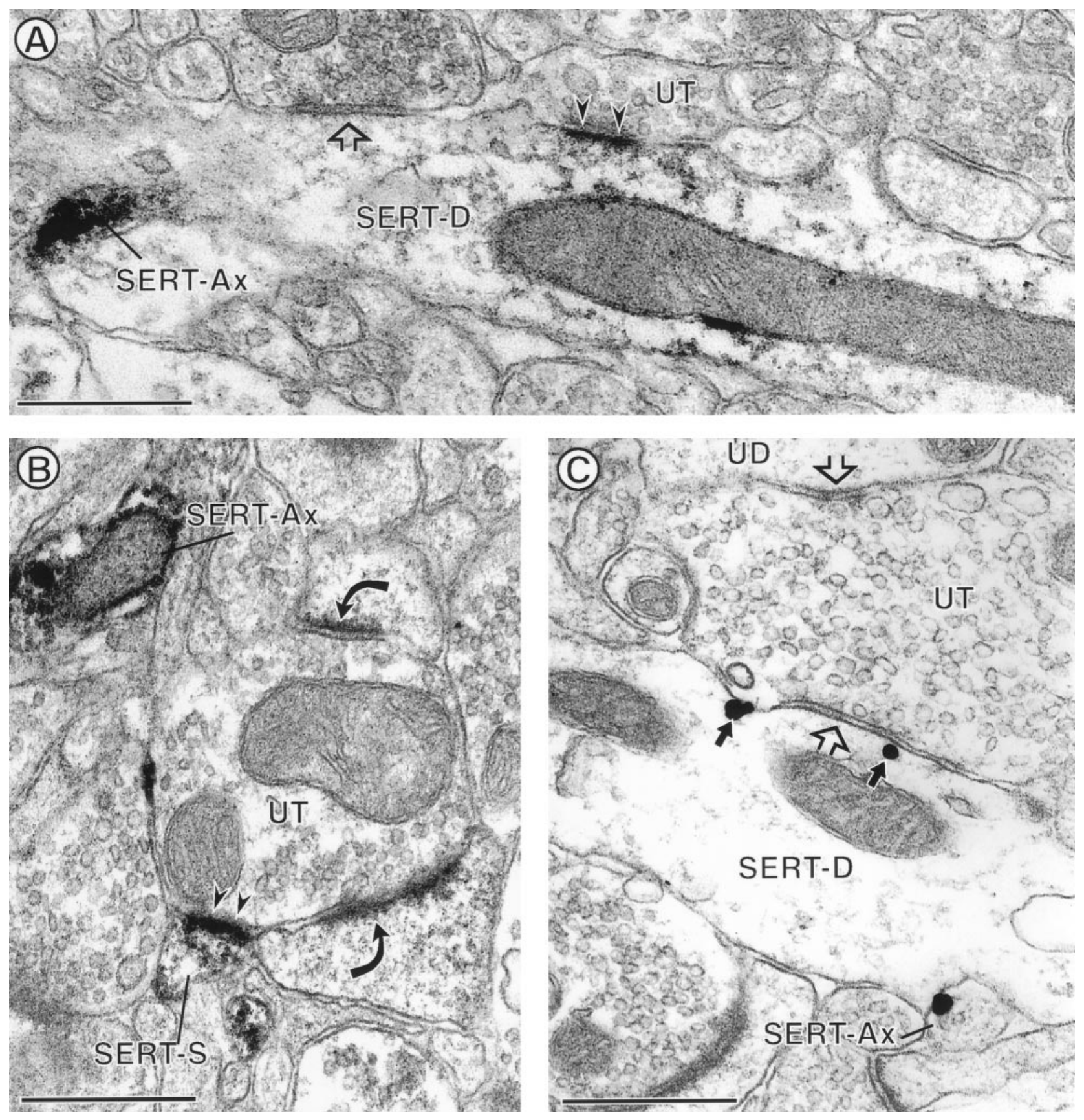

Figure 5. Synaptic plasmalemmal localization of SERT in dendrites (SERT-D) and spines (SERT-S) in the NAc shell by using antisera against amino acids $\mathrm{N}_{1-85}(A, B)$ and $\mathrm{C}_{611-630}(C) . A, B$, Immunoperoxidase reaction product (small arrowheads) for SERT is localized within and near the synapse formed by unlabeled terminals (UT). A, The SERT-D also receives a symmetric synapse (open arrow) from another unlabeled terminal and is apposed to a densely labeled profile resembling an axon $(S E R T-A x)$. B, The unlabeled terminal also provides asymmetric synapses (curved arrows) to two other spines that contain little or no immunoreactivity. $C$, Immunogold-silver labeling (small arrows) in SERT-D is seen within and near the membrane specialization of a symmetric synapse (open arrow) that is formed by an unlabeled terminal (UT). This terminal also forms a similar contact with an unlabeled dendrite $(U D)$ and is apposed to a small SERT-immunolabeled axon (SERT-Ax). Scale bars, $0.5 \mu \mathrm{m}$.

among the presynaptic sites for the antidepressant actions of SERT inhibitors (Nemeroff, 1992).

\section{Nonsynaptic SERT distribution in serotonergic terminals}

Most SERT-immunoreactive axons and terminals in the NAc contained 5-HT in the present dual-labeling study. These terminals are also morphologically similar to previously described
5-HT containing terminals in the NAc (Van Bockstaele and Pickel, 1993). Together, the results show that SERT- and/or 5-HT-containing terminals within the NAc often lack recognizable junctions but sometimes form either asymmetric or symmetric synapses. This heterogeneity may reflect, in part, the functional diversity of postsynaptic 5-HT receptors (Góngora-Alfaro et al., 1997; Pires et al., 1998).

As shown previously in other brain regions (Zhou et al., 1998), 

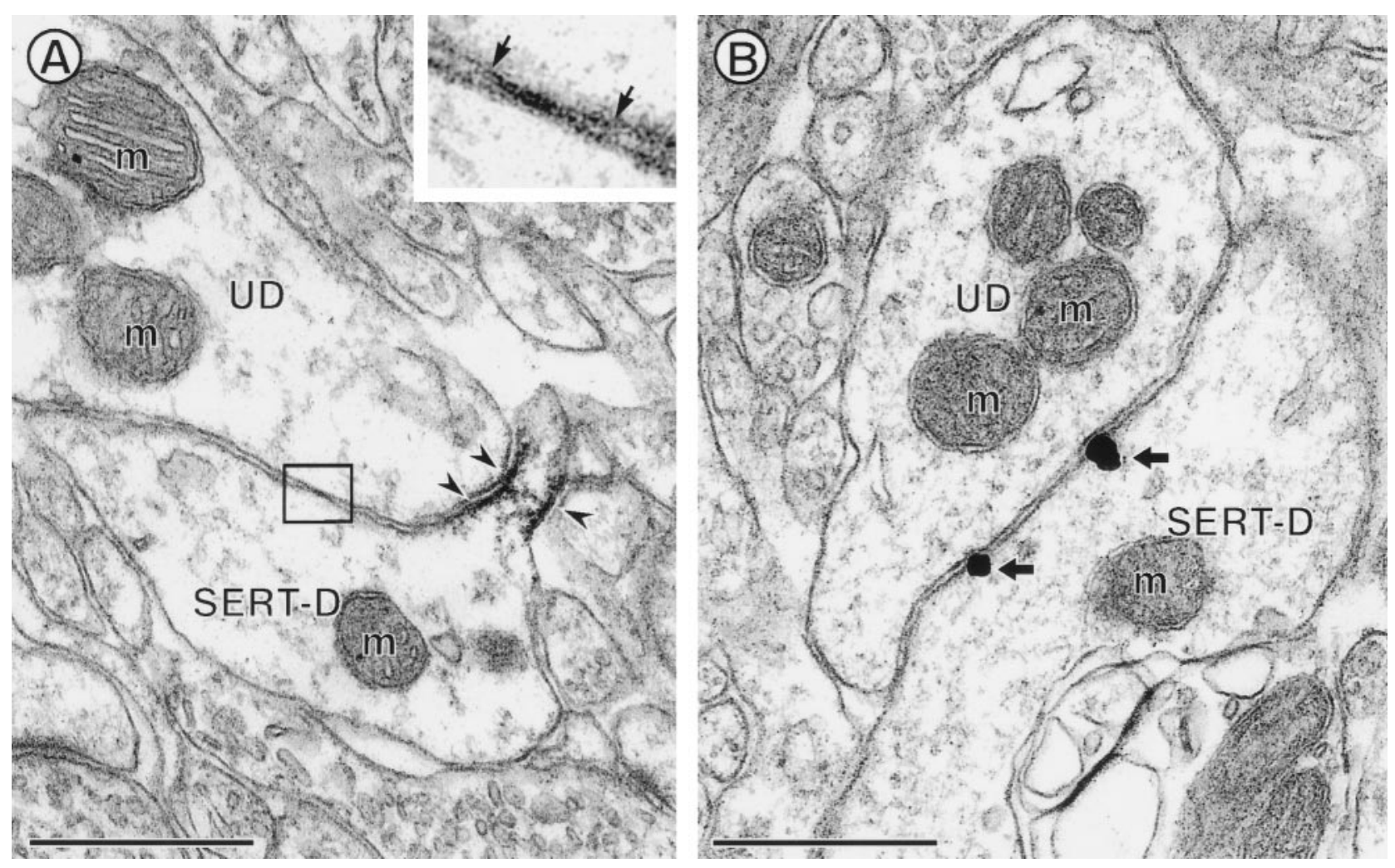

Figure 6. Plasmalemmal localization of SERT in dendrites (SERT-D) apposing other unlabeled dendrites (UD) in the NAc shell. $A$, Immunoperoxidase labeling for mouse $\mathrm{N}_{1-85}$ SERT antiserum at 1:100 dilution in glutaraldehyde-fixed tissue shows reaction product (arrowheads) along apposed and nonapposed surfaces of the plasma membrane. The boxed area in $A$ is shown at a higher magnification in the inset. In the inset the arrows indicate the region in which the inner plasma membranes appear to fuse in a manner typical of gap junctions. $B$, Immunogold labeling for goat anti- $\mathrm{C}_{611-630}$ antiserum at 1:1000 dilution in acrolein-fixed tissue shows immunogold particles (arrows) along the membrane at the appositional contact. In $A$ and $B$ the UD has numerous mitochondria $(m)$, whereas the labeled dendrites have only one within the plane of section. Scale bars, $0.5 \mu \mathrm{m}$.

we rarely saw SERT immunoreactivity within presynaptic membrane specializations in the NAc shell or core. Serotonin is, however, known to be contained within SSVs, some of which are near the active zone of the synapse (Beaudet, 1982; Liem and Copray, 1996). The extrasynaptic distribution of SERT is comparable to that seen with DAT (Nirenberg et al., 1997), suggesting that monoamines diffuse from sites of release into the synaptic cleft to reach more distant receptors in a paracrine mode of transmission (Bunin and Wightman, 1998; Bunin et al., 1998). This hypothesis is consistent with the presence of receptors for 5-HT and dopamine that are located at diverse pre- and postsynaptic, as well as nonsynaptic, sites on plasma membranes (Pickel and Sesack, 1995; Hirst et al., 1998b; Jakab and Goldman-Rakic, 1998).

\section{Dendritic SERT localization}

We observed SERT-LI at selective sites on plasma membranes of a few dendrites in the adult rat NAc. Although SERT mRNA is present in certain nonserotonergic limbic forebrain neurons during development (Hansson et al., 1998), in adult animals the mRNA is seen exclusively in serotonergic neurons of the brainstem raphe in rat (Rattray et al., 1998) and human brain (Austin et al., 1994). The discrepancy between our results and the mRNA studies most likely reflects the greater resolution of electron microscopy as compared with light microscopic in situ hybridization. The presence of SERT in striatal neurons is supported by the fact that we saw similar patterns of immunolabeling by using either of two markers and three different antisera. Furthermore, no labeling was seen in sections that were processed by using peptide-adsorbed SERT antisera. Together, these observations suggest that certain nonserotonergic neurons within the adult NAc express SERT.

In dendrites, SERT-LI was prevalent along portions of the plasma membranes near synaptic contacts, suggesting involvement in the regulation of membrane excitability via voltagesensitive channels (for review, see Beckman and Quick, 1998). This hypothesis is supported by in vitro studies showing that voltage-sensitive channels are influenced markedly by extracellular serotonin and membrane potential (Mager et al., 1994; Lin et al., 1996; Qian et al., 1997). In addition, we occasionally detected SERT-LI on plasma membranes at dendrodendritic appositions, and SERT labeling has been reported on apposed surfaces of adrenal chromaffin cells (Schroeter et al., 1997). Together, the results suggest a role for SERT in synaptic and nonsynaptic communication.

\section{Astrocytic SERT}

We observed SERT-LI within a few filamentous glial processes in the NAc. These results differs from in situ hybridization and light microscopic immunocytochemical studies indicating that SERT mRNA and protein are present exclusively in serotonergic neurons in rat brain tissue sections (Sur et al., 1996; Rattray et al., 1998). SERT mRNA and monoamine oxidase-A (MAO-A), the enzyme that is mainly responsible for serotonin degradation, are known, however, to be present in cultured astrocytes (Fitzgerald et al., 1990; Hirst et al., 1998a). Serotonin uptake in cultured 

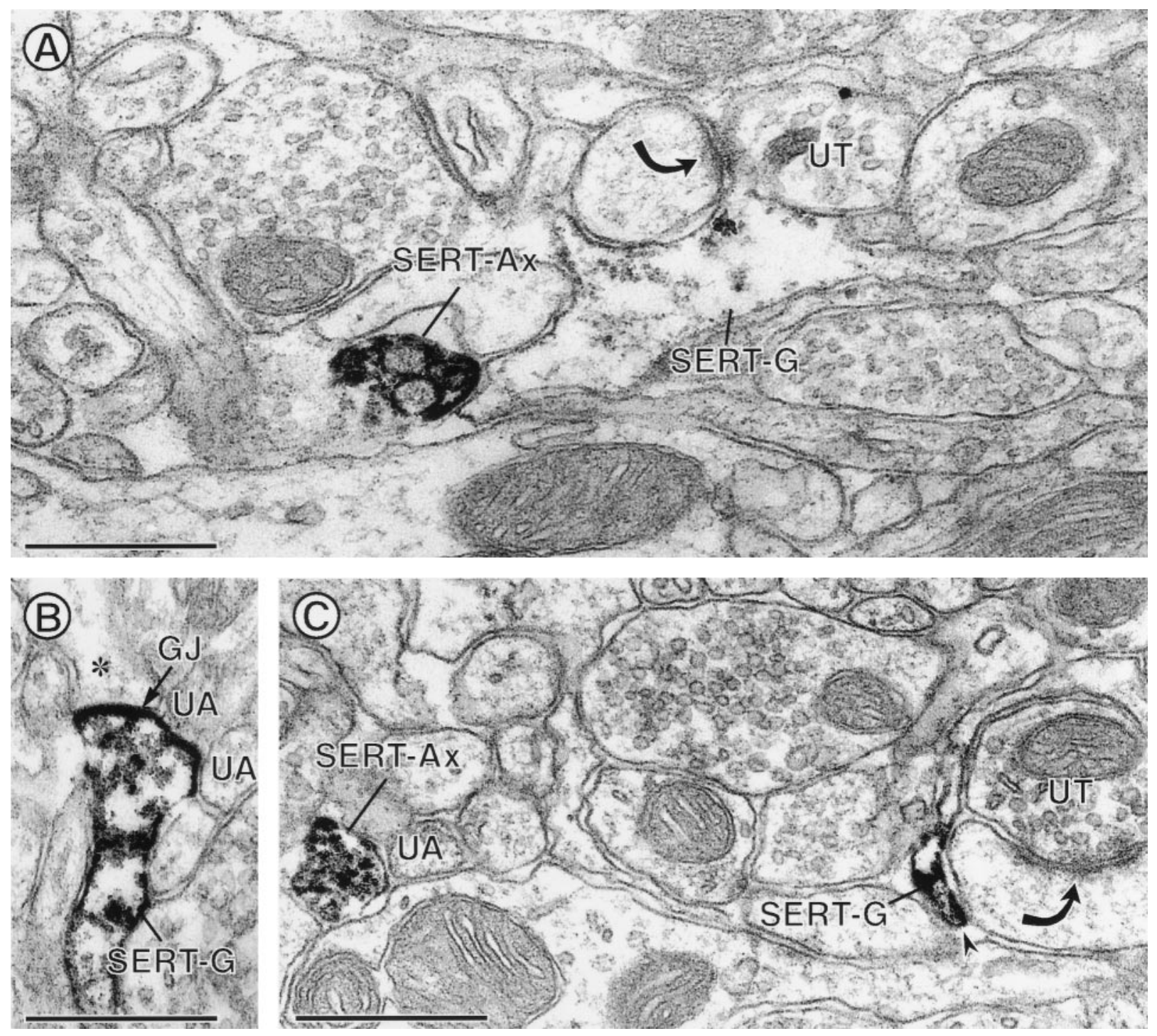

Figure 7. Immunoperoxidase localization of SERT in glial processes (SERT-G) in NAc. Shown is an electron micrograph from the shell region in tissue that was processed by using goat anti- $\mathrm{C}_{611-630}$ antiserum at 1:1000 $(A, C)$ or mouse $\mathrm{N}_{1-85}$ antiserum at 1:100 dilution $(B)$. $A$, The diffuse peroxidase reaction is seen in a glial profile that is apposed to a densely SERT-immunoreactive axon (SERT-Ax) and to an unlabeled terminal (UT) that forms an asymmetric synapse (curved arrow) with a dendrite. $B$, SERT-G shows intense labeling of the plasma membrane at a gap junction $(G J)$ with an unlabeled astrocytic process (asterisk) and at appositional contacts with unlabeled axons $(U A)$. $C$, Micrograph from the NAc core in tissue processed by using goat anti-C $\mathrm{C}_{611-630}$ serum shows SERT-G apposed to an unlabeled terminal (UT) that forms an asymmetric synapse (curved arrow) with an unlabeled dendritic spine. Within the neuropil, intense labeling is seen in an axon (SERT-Ax) apposing an unlabeled axon (UA) and other neuronal profiles. Scale bars, $05 \mu \mathrm{m}$.

astrocytes also is blocked by the antidepressant fluoxetine, and the transporter appears to be similar, if not identical, to SERT in neurons (Fitzgerald et al., 1990; Dave and Kimelberg, 1994; Bal et al., 1997; Hirst et al., 1998b). Together, these results suggest that, in vivo, astrocytes also play a role in the uptake of serotonin in the NAc and possibly other brain regions in which low levels of SERT expression have limited light microscopic detection of mRNA and protein.

\section{Regional comparison of SERT distribution in NAc shell and core}

We have shown that in the NAc shell, as compared with the core, SERT-labeled axon terminals are larger, and axon terminals also contain more serotonin-immunoreactive DCVs. Both size and content of vesicles suggest that the terminals in the shell have greater potential for tonic activity and transmitter release (Lnenicka et al., 1991). In addition, we saw a higher numerical density of SERT-immunoreactive axons and terminals in the NAc shell than in the core but no regional differences in total or plasmalemmal SERT gold particles in individual axonal profiles. Together, these results suggest that greater potential for 5-HT release without a concomitant increase in reuptake may account in large part for the reported higher concentrations of serotonin in the NAc shell versus the core (Deutch and Cameron, 1992). Availability of serotonin (Blakely et al., 1998) as well as regional differences in cytoarchitecture (Meredith et al., 1992; O'Donnell and Grace, 1993) may have contributed to the presently observed lower abundance of SERT-immunoreactive dendrites in the NAc shell as compared with the core. 


\section{REFERENCES}

Austin MC, Bradley CC, Mann JJ, Blakely RD (1994) Expression of serotonin transporter messenger RNA in the human brain. J Neurochem 62:2362-2367.

Bal N, Figueras G, Vilaro MT, Sunol C, Artigas F (1997) Antidepressant drugs inhibit a glial 5-hydroxytryptamine transporter in rat brain. Eur J Neurosci 9:1728-1738.

Beaudet A (1982) High resolution radioautography of central 5-hydroxytryptamine (5-HT) neurons. J Histochem Cytochem 9:765-768.

Beckman ML, Quick MW (1998) Neurotransmitter transporters: regulators of function and functional regulation. J Membr Biol 164:1-10.

Blakely RD, Ramamoorthy S, Schroeter S, Qian Y, Apparsundaram S, Galli A, DeFelice LJ (1998) Regulated phosphorylation and trafficking of antidepressant-sensitive serotonin transporter proteins. Biol Psychiatry 44:169-178.

Brog JS, Salyapongse A, Deutch AY, Zahm DS (1993) The patterns of afferent innervation of the core and shell in the "accumbens" part of the rat ventral striatum: immunohistochemical detection of retrogradely transported fluoro-gold. J Comp Neurol 338:255-278.

Bruns D, Jahn R (1995) Real-time measurement of transmitter release from single synaptic vesicles. Nature 377:62-65.

Bunin MA, Wightman RM (1998) Quantitative evaluation of 5-hydroxytryptamine (serotonin) neuronal release and uptake: an investigation of extrasynaptic transmission. J Neurosci 18:4854-4860.

Bunin MA, Prioleau C, Mailman RB, Wightman RM (1998) Release and uptake rates of 5-hydroxytryptamine in the dorsal raphe and substantia nigra reticulata of the rat brain. J Neurochem 70:1077-1087.

Chan J, Aoki C, Pickel VM (1990) Optimization of differential immunogold-silver and peroxidase labeling with maintenance of ultrastructure in brain sections before plastic embedding. J Neurosci Methods 33:113-127.

Charnay Y, Léger L, Vallet PG, Greggio B, Hof PR, Jouvet M, Bouras C (1996) Mapping of serotonin transporter messenger RNA-containing nerve cell populations in the cat brainstem. J Chem Neuroanat 10:93-100.

Dave V, Kimelberg HK (1994) $\mathrm{Na}^{+}$-dependent, fluoxetine-sensitive serotonin uptake by astrocytes tissue-printed from rat cerebral cortex. J Neurosci 14:4972-4986.

Deutch AY, Cameron DS (1992) Pharmacological characterization of dopamine systems in the nucleus accumbens core and shell. Neuroscience 46:49-56.

Fitzgerald LW, Kaplinsky L, Kimelberg HK (1990) Serotonin metabolism by monoamine oxidase in rat primary astrocyte cultures. J Neurochem 55:2008-2014.

Gobbi M, Parazzoli A, Mennini T (1998) In vitro studies on the mechanism by which $(+)$-norfenfluramine induces serotonin and dopamine release from the vesicular storage pool. Naunyn Schmiedebergs Arch Pharmacol 358:323-327.

Góngora-Alfaro JL, Hernández-López S, Flores-Hernández J, Galarraga E (1997) Firing frequency modulation of substantia nigra reticulata neurons by 5-hydroxytryptamine. Neurosci Res 29:225-231.

Groenewegen HJ, Wright CI, Uylings HBM (1997) The anatomical relationships of the prefrontal cortex with limbic structures and the basal ganglia. J Psychopharmacol 11:99-106.

Gundersen V, Ottersen OP, Storm-Mathisen J (1996) Selective excitatory amino acid uptake in glutamatergic nerve terminals and in glia in the rat striatum: quantitative electron microscopic immunocytochemistry of exogenous D-aspartate and endogenous glutamate and GABA. Eur J Neurosci 8:758-765.

Hansson SR, Mezey É, Hoffman BJ (1998) Serotonin transporter messenger RNA in the developing rat brain: early expression in serotonergic neurons and transient expression in nonserotonergic neurons. Neuroscience 83:1185-1201.

Heidbreder C, Feldon J (1998) Amphetamine-induced neurochemical and locomotor responses are expressed differentially across the anteroposterior axis of the core and shell subterritories of the nucleus accumbens. Synapse 29:310-322.

Hirst WD, Cheung NY, Rattray M, Price GW, Wilkin GP (1998a) Cultured astrocytes express messenger RNA for multiple serotonin receptor subtypes, without functional coupling of 5- $\mathrm{HT}_{1}$ receptor subtypes to adenylyl cyclase. Mol Brain Res 61:90-99.

Hirst WD, Price GW, Rattray M, Wilkin GP (1998b) Serotonin transporters in adult rat brain astrocytes revealed by $\left[{ }^{3} \mathrm{H}\right] 5-\mathrm{HT}$ uptake into glial plasmalemmal vesicles. Neurochem Int 33:11-22.
Jakab RL, Goldman-Rakic PS (1998) 5-Hydroxytryptamine ${ }_{2 \mathrm{~A}}$ serotonin receptors in the primate cerebral cortex: possible site of action of hallucinogenic and antipsychotic drugs in pyramidal cell apical dendrites. Proc Natl Acad Sci USA 95:735-740.

Jones SR, O’Dell SJ, Marshall JF, Wightman RM (1996) Functional and anatomical evidence for different dopamine dynamics in the core and shell of the nucleus accumbens in slices of rat brain. Synapse 23:224-231.

Joyce JN, Goldsmith SG, Gurevich EV (1997) Limbic circuits and monoamine receptors: dissecting the effects of antipsychotics from disease processes. J Psychiatr Res 31:197-217.

Leranth C, Pickel VM (1989) Electron microscopic pre-embedding double immunostaining methods. In: Neuroanatomical tract-tracing methods 2: recent progress (Heimer L, Zaborszky L, eds), pp 129-172. New York: Plenum.

Liem RSB, Copray JCVM (1996) Immunogold localization of serotonin within synaptic terminals in the rat mesencephalic trigeminal nucleus. Acta Anat (Basel) 155:50-56.

Lin F, Lester HA, Mager S (1996) Single-channel currents produced by the serotonin transporter and analysis of a mutation affecting ion permeation. Biophys J 71:3126-3135.

Lnenicka GA, Hong SJ, Combatti M, LePage S (1991) Activitydependent development of synaptic varicosities at crayfish motor terminals. J Neurosci 11:1040-1048.

Mager S, Min C, Henry DJ, Chavkin C, Hoffman BJ, Davidson N, Lester HA (1994) Conducting states of a mammalian serotonin transporter. Neuron 12:845-859.

Meredith GE, Agolia R, Arts MPM, Groenewegen HJ, Zahm DS (1992) Morphological differences between projection neurons of the core and shell in the nucleus accumbens of the rat. Neuroscience 50:149-162.

Mijnster MJ, Raimundo AGV, Koskuba K, Klop H, Docter GJ, Groenewegen HJ, Voorn P (1997) Regional and cellular distribution of serotonin 5-hydroxytryptamine ${ }_{2 a}$ receptor mRNA in the nucleus accumbens, olfactory tubercle, and caudate putamen of the rat. J Comp Neurol 389:1-11.

Muramatsu M, Lapiz MD, Tanaka E, Grenhoff J (1998) Serotonin inhibits synaptic glutamate currents in rat nucleus accumbens neurons via presynaptic 5-HT 1 receptors. Eur J Neurosci 10:2371-2379.

Nemeroff CB (1992) The presynaptic serotonin uptake site in depression. Clin Neuropharmacol 15[Suppl 1]:347A-348A.

Nirenberg MJ, Liu YJ, Peter D, Edwards RH, Pickel VM (1995) The vesicular monoamine transporter 2 is present in small synaptic vesicles and preferentially localizes to large dense core vesicles in rat solitary tract nuclei. Proc Natl Acad Sci USA 92:8773-8777.

Nirenberg MJ, Chan J, Pohorille A, Vaughan RA, Uhl GR, Kuhar MJ, Pickel VM (1997) The dopamine transporter: comparative ultrastructure of dopaminergic axons in limbic and motor compartments of the nucleus accumbens. J Neurosci 17:6899-6907.

Nutt D (1995) The anxiety factor in depression. J Psychopharmacol [Suppl] 9:185-189.

O'Donnell P, Grace AA (1993) Physiological and morphological properties of accumbens core and shell neurons recorded in vitro. Synapse 13:135-160.

Paxinos G, Watson C (1986) The rat brain in stereotaxic coordinates. New York: Academic.

Pelletier G, Steinbusch HW, Verhofstad AAJ (1981) Immunoreactive substance $\mathrm{P}$ and serotonin present in the same dense core vesicle. Nature 293:71-72.

Peters A, Palay SL, Webster HD (1991) The fine structure of the nervous system. New York: Oxford UP.

Pickel VM, Sesack SR (1995) Electron microscopy of central dopamine systems. In: Psychopharmacology (Bloom FE, Kupfer DJ, eds), pp 257-268. New York: Raven.

Pires JG, Silva SR, Ramage AG, Futuro-Neto HD (1998) Evidence that 5 - $\mathrm{HT}_{3}$ receptors in the nucleus tractus solitarius and other brainstem areas modulate the vagal bradycardia evoked by activation of the von Bezold-Jarisch reflex in the anesthetized rat. Brain Res 791:229-234.

Qian Y, Melikian HE, Rye DB, Levey AI, Blakely RD (1995) Identification and characterization of antidepressant-sensitive serotonin transporter proteins using site-specific antibodies. J Neurosci 15:1261-1274.

Qian Y, Galli A, Ramamoorthy S, Risso S, DeFelice LJ, Blakely RD (1997) Protein kinase C activation regulates human serotonin transporters in HEK-293 cells via altered cell surface expression. J Neurosci 17:45-57.

Rattray M, Michael GJ, Lee J, Wotherspoon G, Bendotti C, Priestley JV 
(1998) Intraregional variation in expression of serotonin transporter messenger RNA by 5-hydroxytryptamine neurons. Neuroscience 88:169-183.

Schloss P, Williams DC (1998) The serotonin transporter: a primary target for antidepressant drugs. J Psychopharmacol 12:115-121.

Schroeter S, Levey AI, Blakely RD (1997) Polarized expression of the antidepressant-sensitive serotonin transporter in epinephrinesynthesizing chromaffin cells of the rat adrenal gland. Mol Cell Neurosci 9:170-184.

Schwarting RK, Thiel CM, Müller CP, Huston JP (1998) Relationship between anxiety and serotonin in the ventral striatum. NeuroReport 9:1025-1029.

Sesack SR, Pickel VM (1992) Prefrontal cortical efferents in the rat synapse on unlabeled neuronal targets of catecholamine terminals in the nucleus accumbens septi and on dopamine neurons in the ventral tegmental area. J Comp Neurol 320:145-160.

Staley JK, Malison RT, Innis RB (1998) Imaging of the serotonergic system: interactions of neuroanatomical and functional abnormalities of depression. Biol Psychiatry 44:534-549.
Steinbusch HWM, Verhofstad AAJ, Joosten HWJ (1978) Localization of serotonin in the central nervous system by immunohistochemistry: description of a specific and sensitive technique and some applications. Neuroscience 3:811-819.

Sterio DC (1984) The unbiased estimation of the number and sizes of arbitrary particles using the disector. J Microsc 134:127-136.

Sur C, Betz H, Schloss P (1996) Immunocytochemical detection of the serotonin transporter in rat brain. Neuroscience 73:217-231.

Van Bockstaele EJ, Pickel VM (1993) Ultrastructure of serotoninimmunoreactive terminals in the core and shell of the rat nucleus accumbens: cellular substrates for interactions with catecholamine afferents. J Comp Neurol 334:603-617.

Van Bockstaele EJ, Biswas A, Pickel VM (1993) Topography of serotonin neurons in the dorsal raphe nucleus that send axon collaterals to the rat prefrontal cortex and nucleus accumbens. Brain Res 624:188-198.

Zhou FC, Tao-Cheng JH, Segu L, Patel T, Wang Y (1998) Serotonin transporters are located on the axons beyond the synaptic junctions: anatomical and functional evidence. Brain Res 805:241-254. 